\title{
Common variants of NFE2L2 gene predisposes to acute respiratory distress syndrome in patients with severe sepsis
}

\author{
Marialbert Acosta-Herrera ${ }^{1,2,3}$, Maria Pino-Yanes ${ }^{1,2}$, Jesús Blanco ${ }^{1,4}$, Juan Carlos Ballesteros ${ }^{5}$, Alfonso Ambrós 6 , \\ Almudena Corrales ${ }^{1,2}$, Francisco Gandía ${ }^{7}$, Carlés Subirá ${ }^{8}$, David Domínguez ${ }^{9}$, Aurora Baluja ${ }^{10}$, José Manuel Añón ${ }^{11}$, \\ Ramón Adalia ${ }^{12}$, Lina Pérez-Méndez ${ }^{1,2}$, Carlos Flores ${ }^{1,2,13^{*}}$, and Jesus Villar ${ }^{1,3,14^{*}}$ for the GRECIA and GEN-SEP networks
}

\begin{abstract}
Introduction: The purpose of this study was to investigate whether common variants across the nuclear factor erythroid 2-like 2 (NFE2L2) gene contribute to the development of the acute respiratory distress syndrome (ARDS) in patients with severe sepsis. NFE2L2 is involved in the response to oxidative stress, and it has been shown to be associated with the development of ARDS in trauma patients.

Methods: We performed a case-control study of 321 patients fulfilling international criteria for severe sepsis and ARDS who were admitted to a Spanish network of post-surgical and critical care units, as well as 871 population-based controls. Six tagging single-nucleotide polymorphisms (SNPS) of NFE2L2 were genotyped, and, after further imputation of additional 34 SNPs, association testing with ARDS susceptibility was conducted using logistic regression analysis.
\end{abstract}

Results: After multiple testing adjustments, our analysis revealed 10 non-coding SNPs in tight linkage disequilibrium $\left(0.75 \leq r^{2} \leq 1\right)$ that were associated with ARDS susceptibility as a single association signal. One of those SNPs (rs672961) was previously associated with trauma-induced ARDS and modified the promoter activity of the NFE2L2 gene, showing an odds ratio of 1.93 per T allele (95\% confidence interval, 1.17-3.18; $p=0.0089$ ).

Conclusions: Our findings support the involvement of NFE2L2 gene variants in ARDS susceptibility and reinforce further exploration of the role of oxidant stress response as a risk factor for ARDS in critically ill patients.

\section{Introduction}

Acute respiratory distress syndrome (ARDS) remains a major cause of death in adult intensive care units (ICUs), with most epidemiological reports mentioning a hospital mortality rate over $40 \%$ [1]. Despite a similar pulmonary response, this complex syndrome develops as a complication of several acute disease processes, with sepsis being the most common predisposing condition [1, 2]. Damage to the alveolar-capillary membrane results in increased vascular permeability and protein-rich alveolar edema. The clinical diagnosis is made on the basis of a combination of severe hypoxemia requiring mechanical

\footnotetext{
*Correspondence: cflores@ull.edu.es; jesus.villar54@gmail.com

'CIBER de Enfermedades Respiratorias, Instituto de Salud Carlos III, Madrid, Spain
} Full list of author information is available at the end of the article ventilation with high concentrations of oxygen, bilateral pulmonary infiltrates on chest radiographs, and reduced lung compliance [3].

Critical illness is characterized by an increased production of reactive oxygen species (ROS) [4]. Under physiological conditions, oxygen metabolism generates small amounts of ROS, although the cells have several antioxidant mechanisms against oxidative damage. A disruption of oxidant-antioxidant balance is likely to play a role in the pathogenesis of several inflammatory conditions, including sepsis and ARDS [5]. The nuclear factor erythroid 2-like 2, also known as NRF2 or NFE2L2, plays a central role in the antioxidant mechanisms against ROS. NFE2L2 is a member of the Cap'n'Collar basic leucine zipper transcription factor family and constitutes a hub, controlling the expression of several genes involved in 
regulating cellular antioxidant levels and detoxification [6]. The NFE2L2 gene maps onto chromosome 2 at 2q31. Upon activation by an increase in cellular levels of ROS, NFE2L2 translocates to the nucleus and binds to the antioxidant response element (ARE), inducing the transcription of NFE2L2-regulated genes [6]. In previous positional cloning studies in experimental animals, researchers have identified NFE2L2 as a candidate gene for hyperoxia-induced lung injury susceptibility $[7,8]$. These results were validated in humans, in whom common single-nucleotide polymorphisms (SNPs) were identified by resequencing analysis and candidate SNP functionality was proven in cell lines. In addition, the association of common variants with ARDS susceptibility and mortality has been reported recently [9-11].

In the present study, we aimed to assess the association of common genetic variants in NFE2L2 with ARDS in patients admitted with severe sepsis in a Spanish network of post-surgical and critical care units.

\section{Methods}

This study is part of an ongoing research program in which the role of genetic factors on ARDS susceptibility is being analyzed. This study was approved by the external scientific committee and advisory committee of experts on ethical, economic, environmental, legal, and social affairs at the Spanish national DNA biobank (National DNA Bank Carlos III); the ethics committee at the coordinating center (Hospital Universitario Nuestra Señora de Candelaria, Tenerife, Spain); and the institutional review boards of participating hospitals (Hospital Clínico de Santiago de Compostela, Hospital General de León, Hospital Universitario Río Hortega, Fundació Althaia, Hospital Clinic de Barcelona, Hospital NS del Prado, Hospital Vírgen de la Luz, and Hospital General de Ciudad Real). Informed consent was obtained from all subjects or from their appropriate surrogates.

\section{Study design}

We used a case-control study design with 1222 DNA samples from unrelated individuals. We enrolled 322 patients with a diagnosis of severe sepsis [12] and ARDS who were admitted into a multidisciplinary network of post-surgical and ICUs in Spain (see Appendix). All patients were mechanically ventilated. ARDS was defined according to the Berlin criteria [13]. For the purpose of this study, patients with mild, moderate, and severe ARDS were analyzed as a single group of patients with ARDS. Although the selection of controls remains a challenge [14], we preferred to use population-based subjects as controls instead of using patients at risk, because the former minimize the introduction of selection and Berkson bias $[15,16]$ without sacrificing genotype compliance with Hardy-Weinberg equilibrium (HWE) expectations and therefore provide an additional quality control on genotyping. The population-based control group included DNA samples from 900 unrelated adults (control/case ratio of approximately 3 ) provided by the Spanish national DNA biobank [17]. A health survey was obtained from all control subjects, and none of them had a history of respiratory diseases.

We recorded basic demographic data, severity of illness scores, and clinical information, including source of infection and development of organ failure until ICU discharge. Blood samples for genotyping analysis were collected within the first 24 hours of meeting the criteria for severe sepsis.

\section{Genotyping}

Genomic DNA was extracted from whole blood using an illustra GFX PCR DNA kit (GE Healthcare Life Sciences, Little Chalfont, UK). We followed current guidelines for DNA polymorphism association studies [18]. Sample size was based on an a priori power calculation with Quanto software (http://biostats.usc.edu/Quanto. html) [19] to attain $80 \%$ power for an allele frequency of $10 \%$ and an effect size (odds ratio [OR]) of 1.5, assuming an ARDS incidence of 7.2 new cases per 100,000 population per year in the Spanish population [3].

We first selected a set of six tagging SNPs (tSNPs) using TagIT software [20]. This approach provided a mean coverage of $r^{2}>0.85$ for the common gene variation [minor allele frequency (MAF) $\geq 5 \%$ ] based on the information on the European population derived from the 1000 Genomes Project (1KGP) [21]. Genotyping was performed using the MassARRAY iPLEX Gold ${ }^{\text {mit }}$ platform (Sequenom, San Diego, CA USA) and TaqMan ${ }^{\mathrm{Tx}}$ allelic discrimination assays (Applied Biosystems, Foster City, CA, USA). Individual SNP genotype calls were automatically generated using Sequenom TYPER $3.4^{\mathrm{ma}}$ software. TaqMan genotyping was used for the SNP rs6706649, and performed using a 7500 Fast Real-Time PCR System (Life Technologies, Carlsbad, CA, USA). Genotyping was done blinded to control and case status. DNA from two HapMap individuals and approximately $7 \%$ of the samples was genotyped in duplicate to monitor genotyping quality. The estimated overall genotype concordance among duplicates was $100 \%$ (95\% confidence interval [CI], 95.0-100\%). Twenty-nine control subjects and one patient with ARDS were excluded from downstream analyses because of a low completion rate $(<80 \%)$.

\section{Statistical analysis}

Clinical and demographic data were analyzed with $x^{2}$ tests for categorical variables and the Mann-Whitney $U$ test for ordinal data using R 3.01 software [22]. Quality control and deviations from HWE in genotyped SNPs were assessed using SNPing software [23]. SNP imputation 
using data from European individuals from 1KGP phase I (May 2011) [21] was performed using MaCH 1.0 software [24]. Association testing was conducted for allele dosages using Mach2dat [24] for those SNPs showing MAF $\geq 5 \%$ and squared correlation between imputed and observed genotypes $\left(R^{2}\right) \geq 0.3$. The independence of SNP associations was examined with conditional regression analysis using the $\mathrm{R}$ statistical software package. To control for type I errors arising from multiple hypothesis testing, a false discovery rate (FDR) was calculated by means of qvalue [25]. A FDR threshold of 0.05 was established to declare significance. Pairwise $r^{2}$ values were calculated using Haploview 3.32 [26] to assess the linkage disequilibrium (LD) between SNPs based on data deposited for Europeans in the 1KGP database. Evaluation of functionality of associated SNPs was performed with the online software
HaploReg v3 [27] on the basis of empirical data from the ENCODE project [28] with the aim of identifying functional elements in the human genome sequence. Specifically, we focused our attention on ENCODE experiments performed on cell lines and tissues obtained from lungs, lung developmental stages, and endothelium.

\section{Results}

\section{Characteristics of patients}

Demographic and clinical data from the 321 ARDS patients and 871 population-based control subjects with SNP completion rate $\geq 80 \%$ are summarized in Table 1 . The overall mortality rate at discharge from the ICU was $36.3 \%$. In $35 \%$ of patients, no pathogens were identified as the causative microorganism for sepsis, although all of them had an identified or highly suspected site of

Table 1 Demographic and clinical characteristics of the study sample

\begin{tabular}{|c|c|c|c|c|}
\hline \multicolumn{2}{|l|}{ Characteristic } & $\begin{array}{l}\text { ARDS patients } \\
(n=321)\end{array}$ & $\begin{array}{l}\text { Controls } \\
(n=871)\end{array}$ & $p$ value \\
\hline \multicolumn{2}{|l|}{ Sex (\% male) } & 63.2 & 59.6 & $0.242^{a}$ \\
\hline \multicolumn{2}{|l|}{ Median age, yr $\left(\mathrm{P}_{25}-\mathrm{P}_{75}\right)$} & $67(55-75)$ & $41(32-49)$ & $<0.001^{\mathrm{b}}$ \\
\hline \multicolumn{2}{|l|}{ Hypertension (\%) } & 42.4 & 3.1 & $<0.001^{\mathrm{a}}$ \\
\hline \multicolumn{2}{|l|}{ Smoker (\%) } & 27.9 & 31.2 & $0.422^{a}$ \\
\hline \multicolumn{2}{|l|}{ Previous surgery (\%) } & 65 & NA & \\
\hline \multicolumn{2}{|l|}{ Ischemic cardiac disease (\%) } & 9.0 & NA & \\
\hline \multicolumn{5}{|l|}{ Source of sepsis (\%) } \\
\hline & Pulmonary & 41.9 & \multicolumn{2}{|l|}{ NA } \\
\hline & Extrapulmonary & 58.1 & \multicolumn{2}{|l|}{ NA } \\
\hline \multicolumn{5}{|l|}{ Pathogen (\%) } \\
\hline & Gram-negative & 29.3 & \multicolumn{2}{|l|}{ NA } \\
\hline & Gram-positive & 21.1 & \multicolumn{2}{|l|}{ NA } \\
\hline & Mixed & 5.7 & \multicolumn{2}{|l|}{ NA } \\
\hline & Polymicrobial & 3.3 & \multicolumn{2}{|l|}{ NA } \\
\hline & Virus & 2.8 & \multicolumn{2}{|l|}{ NA } \\
\hline & Fungi & 2.8 & \multicolumn{2}{|l|}{ NA } \\
\hline & Negative blood cultures & 35.0 & \multicolumn{2}{|l|}{ NA } \\
\hline \multicolumn{5}{|l|}{ Organ dysfunction (\%) } \\
\hline & Circulatory & 58.0 & \multicolumn{2}{|l|}{ NA } \\
\hline & Renal & 43.6 & \multicolumn{2}{|l|}{ NA } \\
\hline & Hepatic & 21.4 & \multicolumn{2}{|l|}{ NA } \\
\hline & Neurologic & 20.8 & \multicolumn{2}{|l|}{ NA } \\
\hline & Coagulation & 18.9 & \multicolumn{2}{|l|}{ NA } \\
\hline \multicolumn{2}{|l|}{ APACHE II, mean $\left(P_{25}-P_{75}\right)$} & $22(17-27)$ & \multicolumn{2}{|l|}{ NA } \\
\hline \multicolumn{2}{|l|}{$\mathrm{PaO}_{2} / \mathrm{FiO}_{2}$ mean, ${ }^{\mathrm{c}} \mathrm{mmHg}\left(\mathrm{P}_{25}-\mathrm{P}_{75}\right)$} & \multicolumn{3}{|l|}{$206(124-255)$} \\
\hline \multicolumn{2}{|l|}{ ICU mortality (\%) } & 36.3 & \multicolumn{2}{|l|}{ NA } \\
\hline
\end{tabular}

APACHE II Acute Physiology and Chronic Health Evaluation II, ICU intensive care unit, $N A$ not applicable, $P_{25}$ percentile $25, P_{75}$ percentile $75, P_{a} \mathrm{O}_{2} / F i O_{2}$ ratio of partial pressure arterial oxygen and fraction of inspired oxygen

${ }^{\mathrm{a}} \mathrm{X}^{2}$ test

${ }^{b}$ Mann-Whitney $U$ test

${ }^{c}$ At the time of onset 
infection, a finding that is in accordance with published data [29]. The most common sites of infection were the lung, the abdominal cavity, and the gastrointestinal tract.

\section{Genotype frequencies and association with acute respiratory distress syndrome}

All the tSNPs had a genotype completion rate $>95 \%$. In the control group, none of the six tSNPs deviated significantly from HWE expectations (Table 2). After imputation, association testing was conducted for 40 SNPs with MAF $\geq 5 \%$ and $R^{2} \geq 0.3$. Finally, a total of 10 SNPs, all non-coding, were significantly associated with ARDS susceptibility after multiple testing adjustments $(\mathrm{FDR}=$ 0.036), with the top hit being rs 4243387 (OR for $\mathrm{C}$ allele $=$ 1.93; $95 \%$ CI, 1.19-3.12; $p=0.0068$ ) (Table 3). Although the 10 SNPs associated are distributed along approximately $10 \mathrm{~kb}$ of the gene, and though several of them relate to histone marks according to empirical data (Table 3), they constitute a single association signal, owing to the strong LD among them $\left(0.75 \leq r^{2} \leq 1\right)$ (Fig. 1). In fact, association analyses using regression models accounting for the top hit rendered the remaining SNPs non-significant (Table 4). In addition, of the 10 associated SNPs that were not independent from each other, it is worth noting that the rs6721961 was among the associated SNPs showing an OR of 1.93 per each addition of a T allele (95 \% CI, 1.17-3.18; $p=0.0089$ ). This SNP is located at $-178 \mathrm{bp}$ from the transcription start site of the gene [30] and was previously associated with trauma-induced ARDS, although it was described at position $-617 \mathrm{bp}$ in that publication [9].

\section{Discussion}

This study is the first examining, the association of common variants of NFE2L2 gene with susceptibility to ARDS among patients with severe sepsis, finding an association of 10 SNPs with this syndrome. Although these SNPs were widely distributed across the gene, all of them showed strong LD with each other. One of the associated SNPs, rs6721961, which showed a minor allele frequency of $11.1 \%$ in this sample, was located in the promoter region of the gene, and its T-allele had been previously linked to a reduction in functionality that limited the NFE2L2 triggering of the antioxidative response [9]. Consistent with those findings, we found that the $\mathrm{T}$ allele at rs6721961 conferred greater risk for ARDS susceptibility in patients with sepsis than in healthy subjects. A functional evaluation of the associated SNPs with empirical data from the ENCODE project revealed that seven of them are located in histone marks and/or on DNase I hypersensitivity sites (Table 3). Specifically, the SNP rs6721961 locates in a promoter histone mark in lung fibroblasts and fetal lung. It is also located in a DNase I hypersensitivity site, as reported in an epithelial cell line derived from a lung carcinoma tissue. Given that chromatin modifications on histone marks are critically involved in the regulation of gene expression and that these regions tend to collocate with DNase-sensitive sites in transcriptional start sites [31], this evidence highlights the key role of rs6721961 in the regulation of NFE2L2 expression. Overall, our results highlight the importance of NFE2L2 gene variants in modulating the response to oxidative damage among critically ill patients.

Reduction-oxidation (redox) balance is particularly important in the airways because they represent the first contact with environmental oxidants. Generation of ROS has been implicated in the pathogenesis of many acute and chronic pulmonary diseases, including ARDS [32], and it is a common condition among critically ill patients that results in the development of multiple organ system failure [33]. In this respect, researchers in several studies have reported the presence of oxidative damage in patients with sepsis [34, 35]. High levels of protein oxidation have been found, both in plasma and in bronchoalveolar fluids, in early stages of severe sepsis development and during major trauma [36]. NFE2L2 constitutes a hub and a master regulator of detoxifying systems, such as catalase, superoxide dismutase, and glutathione peroxidase, that are critically involved in protecting the cells against oxidative stress [6]. Using experimental animal models where this transcription factor was disrupted allowed the identification of dependent genes that are critical in pulmonary

Table 2 Location, allele frequency, and quality control information for tagging single-nucleotide polymorphisms genotyped for NFE2L2 gene

\begin{tabular}{lllllll}
\hline tSNPs & Alleles & Position $^{a}$ & CR (\%) & MAF cases & MAF controls & HWE controls \\
\hline rs35652124 & T/C & $177,265,344$ & 98.3 & 0.250 & 0.274 & 0.231 \\
rs6706649 & C/T & $177,265,342$ & 95.2 & 0.115 & 0.126 & 0.341 \\
rs2364722 & A/G & $177,260,058$ & 99.6 & 0.254 & 0.280 & 0.152 \\
rs72946143 & T/C & $177,253,423$ & 100 & 0.044 & 0.067 & 0.270 \\
rs1806649 & C/T & $177,253,423$ & 99.3 & 0.300 & 0.317 & 0.481 \\
rs6726395 & A/G & $177,238,500$ & 99.1 & 0.469 & 0.494 & 0.378 \\
\hline
\end{tabular}

$C R$ completion rate, HWE Hardy-Weinberg equilibrium $p$ value, MAF minor allele frequency

${ }^{a}$ According to National Center for Biotechnology Genome Reference Consortium NCBI build GRCh38 
Table 3 Summary of NFE2L2 variants associated with acute respiratory distress syndrome susceptibility with false discovery rate $<0.05$

\begin{tabular}{|c|c|c|c|c|c|c|c|}
\hline Position $^{a}$ & SNPS & Functionality $^{\mathrm{b}}$ & Minor allele & MAF & $R^{2}$ & OR $(95 \%$ Cl) & $p$ value \\
\hline \multirow[t]{2}{*}{$177,265,308$} & rs6721961 & 5' flanking & $\mathrm{T}$ & 0.111 & 0.32 & $1.93(1.17-3.18)$ & 0.0089 \\
\hline & & Histone mark, DHS & & & & & \\
\hline $177,255,662$ & rs10188193 & Intron 1 & $\mathrm{~T}$ & 0.110 & 0.33 & $1.95(1.19-3.17)$ & 0.0071 \\
\hline $177,255,583$ & rs10188107 & Intron 1 & $\mathrm{~T}$ & 0.110 & 0.33 & $1.95(1.19-3.17)$ & 0.0071 \\
\hline \multirow[t]{2}{*}{$177,254,567$} & rs10497511 & Intron 1 & G & 0.110 & 0.33 & $1.95(1.19-3.17)$ & 0.0070 \\
\hline & & DHS & & & & & \\
\hline \multirow[t]{2}{*}{$177,253,821$} & rs2001297 & Intron 1 & C & 0.110 & 0.33 & $1.95(1.20-3.16)$ & 0.0069 \\
\hline & & Histone mark & & & & & \\
\hline \multirow[t]{2}{*}{$177,253,036$} & rs $4243387^{c}$ & Intron 1 & C & 0.112 & 0.34 & $1.93(1.19-3.12)$ & 0.0068 \\
\hline & & Histone mark & & & & & \\
\hline $177,249,903$ & rs10930781 ${ }^{c}$ & Intron 1 & A & 0.106 & 0.35 & $1.90(1.17-3.12)$ & 0.0085 \\
\hline \multirow[t]{2}{*}{$177,248,755$} & rs1962142 & Intron 1 & A & 0.103 & 0.33 & $1.96(1.18-3.23)$ & 0.0083 \\
\hline & & Histone mark & & & & & \\
\hline \multirow[t]{2}{*}{$177,240,415$} & rs2364720 & Intron 1 & A & 0.106 & 0.35 & $1.90(1.17-3.09)$ & 0.0082 \\
\hline & & Histone mark & & & & & \\
\hline \multirow[t]{2}{*}{$177,235,696$} & rs $2001350^{c}$ & Intron 1 & C & 0.103 & 0.32 & $2.00(1.20-3.35)$ & 0.0075 \\
\hline & & Histone mark & & & & & \\
\hline
\end{tabular}

Cl confidence interval, DHS DNase I hypersensitivity site, FDR false discovery rate, MAF minor allele frequency, OR odds ratio, $R^{2}$ squared correlation between imputed and observed genotypes, SNP single-nucleotide polymorphism

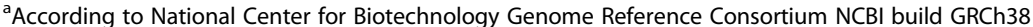

${ }^{b}$ Functionality obtained from HaploReg v3 [27]

'SNPs associated with primary graft dysfunction in Cantu et al. [11]

protection and confirmed that its disruption promotes susceptibility to several prooxidant-induced lung diseases, primarily owing to decreased levels of the basal and inducible expression of several antioxidant enzymes [37-40].

The importance of redox balance in the pathogenesis of ARDS, as well as the implication of NFE2L2 in disease susceptibility or outcome, is supported by the identification of variants in a few genes involved in the oxidative stress response previously associated with ARDS susceptibility or outcome [41, 42]. Marzec et al. [9] assessed the functionality of the SNP rs6721961 (referred to by those authors at position $-617 \mathrm{bp}$ ) on the promoter region by means of a reporter gene assay and an electrophoretic mobility shift assay (EMSA). In the reporter gene assay, the luciferase activity for the $\mathrm{T}$ allele was less than half of the activity for the $G$ allele, indicating a significant reduction of NFE2L2 gene expression. Congruently, results derived from the EMSA showed that the formation of a protein-DNA complex was significantly diminished in the presence of the $\mathrm{T}$ allele, suggesting a less efficient binding of the NFE2L2 transcription factor to the ARE-like sequences of its target genes. In the same study, and consistent with these results, the presence of the T allele at rs6721961 was associated with risk for ARDS susceptibility in a nested case-control association study conducted with 30 patients with trauma- induced ARDS and 60 matched at-risk control subjects. A fixed-effects meta-analysis combining these results with those derived from our study confirmed the concordance of effects at the SNP level, showing an OR for the $\mathrm{T}$ allele of 2.18 (95\% CI, 1.35-3.50; $p=0.0013$ ). The same SNP was recently found to be associated with 28 day mortality in a nested case-control study that included 224 patients with ARDS from a cohort of 750 patients with systemic inflammatory response syndrome [10]. Also, Cantu et al. [11] found a few NFE2L2 SNPs associated with primary graft dysfunction, a specific form of ARDS developed within 72 hours after lung transplantation. Four of those SNPs (rs10930781, rs1962142, rs2001350, and rs4243387) were also significantly associated with ARDS in our study.

The reliability of association findings can be assessed only by replicating the results in independent samples. Such an effort has been widely recognized as a major gap in the field [14, 43]. In this respect, the present study can be considered a SNP-level replication of previous findings in a large series of patients, although the precipitating injury was severe sepsis instead of trauma. As a result of this, although ancestry adjustments were not implemented, a confounder effect due to the presence of population stratification in this study would be minimal. Besides, we acknowledge some minor limitations. 


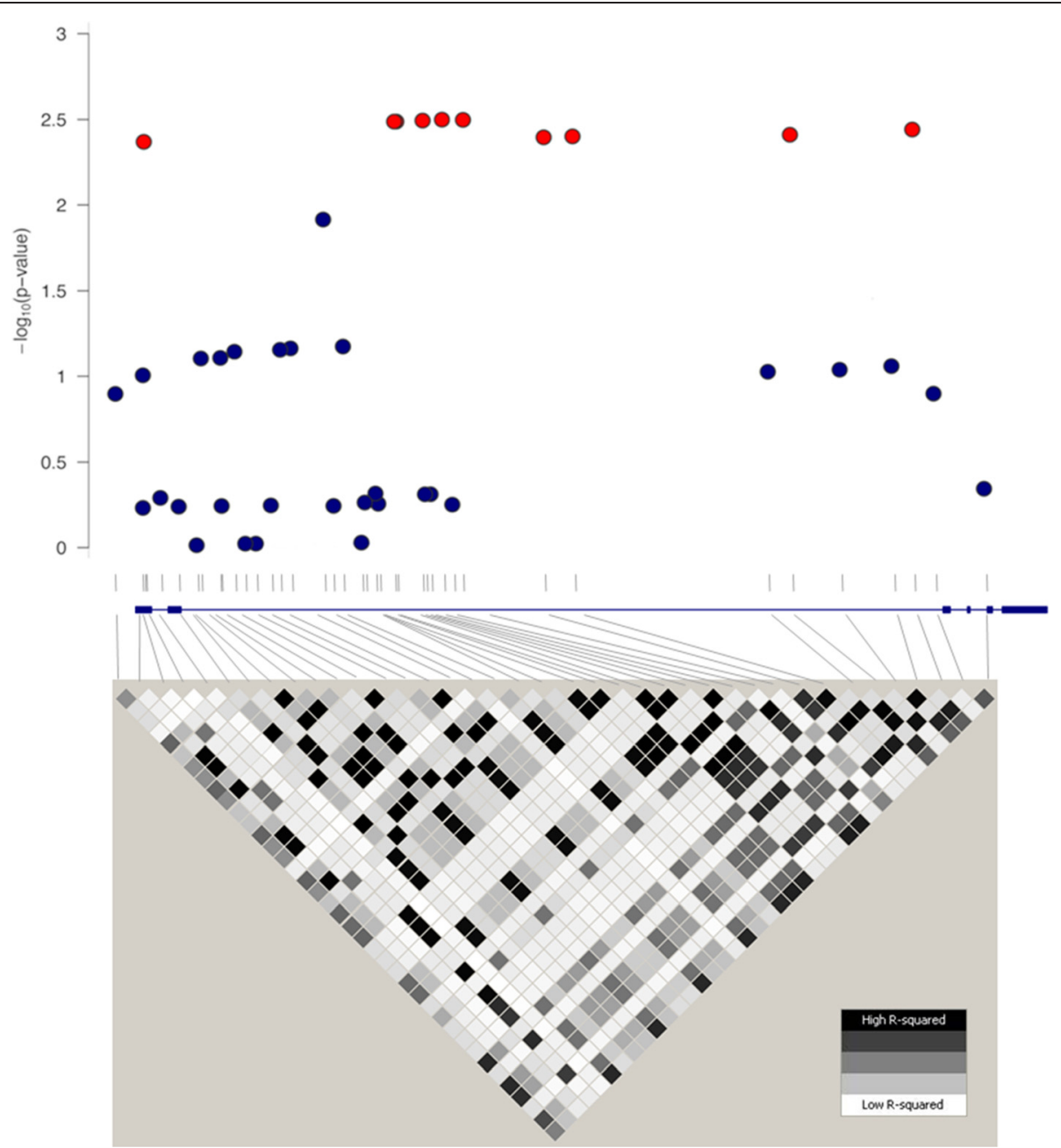

Fig. 1 Regional plot of association results. Upper panel: The $y$-axis represents the $-\log _{10}$-transformed $p$ values for association tests. The $x$-axis represents the approximate location of the 40 single-nucleotide polymorphisms (SNPs) tested for association relative to the gene. Red circles depict associated SNPs after multiple comparison adjustments. Lower panel: This linkage disequilibrium (LD) plot is based on pairwise $r^{2}$ values among SNPs from the European population of the 1000 Genomes Project. Each diamond of the LD plot represents an $r^{2}$ value between two SNPs, schematically symbolized by a color gradient ranging from black $\left(r^{2}=1\right.$, corresponding to complete LD) to gray $\left(1<r^{2}<0\right.$, moderate LD) and white $\left(r^{2}=0\right.$, absence of $\left.L D\right)$

Table 4 Conditional regression results accounting for the effect of rs4243387

\begin{tabular}{lll}
\hline SNP & $\begin{array}{l}\text { Univariate } \\
\text { association } p \text { value }\end{array}$ & $\begin{array}{l}\text { Conditional } \\
\text { regression } p \text { value }\end{array}$ \\
\hline rs10188107 & 0.0071 & 0.464 \\
rs10188193 & 0.0071 & 0.498 \\
rs10497511 & 0.0070 & 0.580 \\
rs10930781 & 0.0085 & 0.929 \\
rs1962142 & 0.0083 & 0.956 \\
rs2001297 & 0.0069 & 0.678 \\
rs2001350 & 0.0075 & 0.940 \\
rs2364720 & 0.0082 & 0.980 \\
rs6721961 & 0.0089 & 0.292 \\
\hline
\end{tabular}

SNP single-nucleotide polymorphism
First, although the study sample provided $80 \%$ power to detect a minimum risk of 1.5 , we recognize that this sample size is limited to detect smaller effect sizes that are expected on average for complex traits [44]. Second, we assessed only the common variation within the NFE2L2 gene, and they would contribute to an explanation of only a modest fraction of the genetic component of the syndrome. Third, the use of populationbased controls instead of at-risk controls precludes deducing whether the NFE2L2 gene is associated with ARDS or with the underlying condition (e.g., severe sepsis). However, whatever the case, the fact that our results replicated previous findings from a study using at-risk controls strongly supports that this gene is directly involved in ARDS susceptibility. 


\section{Conclusions}

We provide evidence implicating common NFE2L2 gene variants in ARDS susceptibility, reinforcing further explorations of the role of oxidant stress response as a risk factor for ARDS in critically ill patients. Research in this field will eventually translate into potentially useful information by identifying new pathways and novel therapeutic approaches, and also by developing predisposition biomarkers to stratify at-risk patients, thus facilitating personalized patient assessment and better patient management.

\section{Key messages}

- A number of common variants of the NFE2L2 gene are associated with ARDS in patients with severe sepsis.

- One of the associated SNPs is located in the promoter region and has been proven to modify the promoter activity of the NFE2L2 gene.

- Our study supports the role of the oxidant stress response as a risk factor for ARDS in critically ill patients, irrespective of the precipitating injury.

\section{Appendix \\ GEN-SEP investigators}

Jesús Villar, Rosa L. Fernández (Hospital Universitario Dr. Negrín, Las Palmas de Gran Canaria); Carlos Flores, Maria Pino-Yanes, Marialbert Acosta-Herrera, Lina Pérez-Méndez, Almudena Corrales, Elena Espinosa, David Domínguez (Hospital Universitario Nuestra Señora de Candelaria, Santa Cruz de Tenerife); Alfonso Ambrós, Rafael del Campo (Hospital General de Ciudad Real, Ciudad Real); Rafael Fernández, Carles Subirá, José A. Rodríguez (Fundació Althaia, Barcelona); Aurora Baluja, Julián Álvarez (Hospital Clínico Universtario de Santiago de Compostela, La Coruña); José M. Añón, Elena González, Oscar Hernández, Rosario Solano, Javier Pérez-Crespo, Paola Arellano (Hospital Virgen de La Luz, Cuenca); Ramón Adalia, Eli Zavala, Julia Martínez, Antoni Torres, Joan Badia (Hospital Clínic, Barcelona); and Francisco Alba, Ruth Corpas (Hospital Nuestra Señora del Prado, Toledo).

\section{GRECIA investigators}

Jesús Blanco, Arturo Muriel (Hospital Universitario Río Hortega, Valladolid); Victor Sagredo, Juan C. Ballesteros (Hospital Clínico Universitario de Salamanca, Salamanca); Francisco Taboada, Guillermo M. Albaiceta (Hospital Central de Asturias, Oviedo); Francisco Gandía, Felipe Bobillo (Hospital Clínico Universitario de Valladolid, Valladolid); Luis Tamayo (Hospital Río Carrión, Palencia); A.G. Labattut (Hospital General de Soria, Soria); Demetrio Carriedo, Javier Collado, Francisco J. Diaz (Hospital
General de León, León); M. Valledor, Maite Antuña (Hospital San Agustín, Avilés); M. de Frutos (Hospital General Yagüe, Burgos); María J. López, José J. Cortina (Hospital General de Segovia, Segovia); Teresa Saldaña, Ana Caballero, Teresa Álvarez (Hospital Virgen de la Concha, Zamora); and Braulio Álvarez, José Sandoval (Hospital del Bierzo, Ponferrada).

\section{Abbreviations}

1KGP: The 1000 genomes project; APACHE II: Acute Physiology and Chronic Health Evaluation II; ARDS: Acute respiratory distress syndrome; ARE: Antioxidant response element; Cl: Confidence interval; CR: Completion rate; DHS: DNase I hypersensitivity site; EMSA: Electrophoretic mobility shift assay; FDR: False discovery rate; HWE: Hardy-Weinberg equilibrium; ICU: Intensive care unit; LD: Linkage disequilibrium; MAF: Minor allele frequency; NFE2L2: Nuclear factor erythroid 2-like 2; OR: Odds ratio; $\mathrm{PaO}_{2} / \mathrm{FiO}_{2}$ : Ratio of partial pressure arterial oxygen and fraction of inspired oxygen; Redox: Reduction-oxidation; ROS: Reactive oxygen species; SNP: Single nucleotide polymorphism; tSNP: Tagging single-nucleotide polymorphism.

\section{Competing interests}

The authors declare that they have no competing interests.

\section{Authors' contributions}

$J V$ and CF conceived and designed the original study. JB, JCB, AA, FG, CS, $D D, A B, J M A$, and RA enrolled patients and collected the data. JV obtained funding for the study. MAH, MPY, AC, and CF carried out the genetic analysis. MAH, MPY, LPM, CF, and JV performed the statistical analysis and interpreted the findings. MAH, MPY, JB, JCB, AA, FG, CS, DD, AB, JMA, RA, AC, LPM, CF, and JV made substantial contributions to the study's conception and design, or acquisition of data, or analysis and interpretation of data, or drafting of the article or revising it critically for important intellectual content. $M A H, M P Y, C F$, and JV wrote the first manuscript draft. MAH, MPY, JB, JCB, AA, $F G, C S, D D, A B, J M A, R A, A C, L P M, C F$, and JV approved the final version to be published. MAH, MPY, LPM, CF, and JV had full access to all data in the study and take responsibility for the integrity of the data and the accuracy of the data analysis. All authors read and approved the final manuscript.

\section{Authors' information}

CF and JV contributed equally as senior authors.

\section{Acknowledgements}

This work was funded by the Instituto de Salud Carlos III (CB06/06/1088, PI10/00393, PI14/00844, FI11/00074) and by the European Regional Development Funds "A way of making Europe." MPY was supported by a post-doctoral fellowship from Fundación Ramón Areces, Madrid, Spain. The funders had no role in the study design, data collection and analysis, decision to publish, or preparation of the manuscript. Members of the GRECIA and GEN-SEP networks are listed in the Appendix.

\section{Author details}

'CIBER de Enfermedades Respiratorias, Instituto de Salud Carlos III, Madrid, Spain. ${ }^{2}$ Research Unit, Hospital Universitario Nuestra Señora de Candelaria, Carretera del Rosario 145, 38010 Santa Cruz de Tenerife, Spain. ${ }^{3}$ Multidisciplinary Organ Dysfunction Evaluation Research Network, Research Unit, Hospital Universitario Dr. Negrín, Barranco de la Ballena s/n - 4th floor, south wing, 35019 Las Palmas de Gran Canaria, Spain. ${ }^{4}$ Intensive Care Unit, Hospital Universitario Río Hortega, Valladolid, Spain. Intensive Care Unit, Hospital Clínico Universitario de Salamanca, Salamanca, Spain. Intensive Care Unit, Hospital General Universitario de Ciudad Real, Ciudad Real, Spain. IIntensive Care Unit, Hospital Clínico de Valladolid, Valladolid, Spain. I'Intensive Care Unit, Fundació ALTHAIA, Manresa, Spain. ${ }^{9}$ Department Anesthesia, Hospital Universitario Nuestra Señora de Candelaria, Santa Cruz de Tenerife, Spain. ${ }^{10}$ Department Anesthesiology, Hospital Clínico Universitario, Santiago de Compostela, Spain. ${ }^{11}$ Intensive Care Unit, Hospital Virgen de La Luz, Cuenca, Spain. ${ }^{12}$ Department of Anesthesiology, Hospital Clinic de Barcelona, Barcelona, Spain. ${ }^{13}$ Applied Genomics Group, Laboratory of Genetics, Instituto Universitario de Enfermedades Tropicales y Salud Pública de Canarias, Universidad de La Laguna, Tenerife, Spain. ${ }^{14}$ Keenan Research Center for Biomedical Science, St. Michael's Hospital, Toronto, ON, Canada. 
Received: 23 March 2015 Accepted: 5 June 2015

Published online: 16 June 2015

\section{References}

1. Villar J, Sulemanji D, Kacmarek RM. The acute respiratory distress syndrome: incidence and mortality, has it changed? Curr Opin Crit Care. 2014;20:3-9.

2. Hudson LD, Milberg JA, Anardi D, Maunder RJ. Clinical risks for development of the acute respiratory distress syndrome. Am J Respir Crit Care Med. 1995;151:293-301.

3. Villar J, Blanco J, Añón JM, Santos-Bouza A, Blanch L, Ambrós A, et al. The ALIEN study: incidence and outcome of acute respiratory distress syndrome in the era of lung protective ventilation. Intensive Care Med. 2011;37:1932-41. A published erratum appears in Intensive Care Med. 2011 Dec;37(12):1942.

4. Abilés J, de la Cruz AP, Castaño J, Rodríguez-Elvira M, Aguayo E, Moreno-Torres $\mathrm{R}$, et al. Oxidative stress is increased in critically ill patients according to antioxidant vitamins intake, independent of severity: a cohort study. Crit Care. 2006;10:R146.

5. Crimi E, Sica V, Williams-Ignarro S, Zhang H, Slutsky AS, Ignarro LJ, et al. The role of oxidative stress in adult critical care. Free Radic Biol Med. 2006:40:398-406.

6. Cho HY, Reddy SP, Kleeberger SR. Nrf2 defends the lung from oxidative stress. Antioxid Redox Sig. 2006;8:76-87.

7. Cho HY, Jedlicka AE, Reddy SP, Zhang LY, Kensler TW, Kleeberger SR. Linkage analysis of susceptibility to hyperoxia: Nrf2 is a candidate gene. Am J Respir Cell Mol Biol. 2002;26:42-51.

8. Cho HY, Jedlicka AE, Reddy SP, Kensler TW, Yamamoto M, Zhang LY, et al. Role of NRF2 in protection against hyperoxic lung injury in mice. Am J Respir Cell Mol Biol. 2002;26:175-82.

9. Marzec JM, Christie JD, Reddy SP, Jedlicka AE, Vuong H, Lanken PN, et al. Functional polymorphisms in the transcription factor NRF2 in humans increase the risk of acute lung injury. FASEB J. 2007;21:2237-46.

10. O'Mahony DS, Glavan BJ, Holden TD, Fong C, Black RA, Rona G, et al. Inflammation and immune-related candidate gene associations with acute lung injury susceptibility and severity: a validation study. PLoS One. 2012; :e51104

11. Cantu E, Shah RJ, Lin W, Daye ZJ, Diamond JM, Suzuki Y, et al. Oxidant stress regulatory genetic variation in recipients and donors contributes to risk of primary graft dysfunction after lung transplantation. J Thorac Cardiovasc Surg. 2015;149:596-602.

12. Levy MM, Fink MP, Marshall JC, Abraham E, Angus D, Cook D, et al. 2001 SCCM/ESICM/ACCP/ATS/SIS International Sepsis Definitions Conference. Crit Care Med. 2003;31:1250-6.

13. Ranieri VM, Rubenfeld GD, Thompson BT, Ferguson ND, Caldwell E, Fan E, et al. The ARDS Definition Task Force. Acute respiratory distress syndrome: the Berlin Definition. JAMA. 2012;307:2526-33.

14. Acosta-Herrera M, Pino-Yanes M, Perez-Mendez L, Villar J, Flores C. Assessing the quality of studies supporting genetic susceptibility and outcomes of ARDS. Front Genet. 2014;5:20.

15. Vineis $P$, McMichael AJ. Bias and confounding in molecular epidemiological studies: special considerations. Carcinogenesis. 1998;19:2063-7.

16. Weiss ST. Association studies in asthma genetics. Am J Respir Crit Care Med. 2001;164:2014-5.

17. Spanish National DNA Bank. http://www.bancoadn.org/. Accessed 19 June 2015

18. Little J, Higgins JP, loannidis JP, Moher D, Gagnon F, von Elm E, et al. STrengthening the REporting of Genetic Association Studies (STREGA): an extension of the STROBE statement. PLoS Med. 2009;6:e22.

19. Gauderman WJ. Sample size requirements for matched case-control studies of gene-environment interaction. Stat Med. 2002;21:35-50.

20. Weale ME, Depondt C, Macdonald SJ, Smith A, Lai PS, Shorvon SD, et al. Selection and evaluation of tagging SNPs in the neuronal-sodium-channel gene SCN1A: implications for linkage-disequilibrium gene mapping. Am J Hum Genet. 2003;73:551-65.

21. Abecasis GR, Auton A, Brooks LD, DePristo MA, Durbin RM, Handsaker RE, et al. An integrated map of genetic variation from 1,092 human genomes. Nature. 2012;491:56-65.

22. R Core Team. R: a language and environment for statistical computing. Vienna; 2008. http://www.R-project.org. Accessed 19 June 2015.

23. Sun X, Ma SF, Wade MS, Flores C, Pino-Yanes M, Moitra J, et al. Functional variants of the sphingosine-1-phosphate receptor 1 gene associate with asthma susceptibility. J Allergy Clin Immunol. 2010;126:241-9.
24. Li Y, Willer CJ, Ding J, Scheet P, Abecasis GR. MaCH: using sequence and genotype data to estimate haplotypes and unobserved genotypes. Genet Epidemiol. 2010;34:816-34.

25. Storey JD, Tibshirani R. Statistical significance for genomewide studies. Proc Natl Acad Sci U S A. 2003;100:9440-5.

26. Barrett JC, Fry B, Maller J, Daly MJ. Haploview: analysis and visualization of LD and haplotype maps. Bioinformatics. 2005;21:263-5.

27. Ward LD, Kellis M. HaploReg: a resource for exploring chromatin states, conservation, and regulatory motif alterations within sets of genetically linked variants. Nucleic Acids Res. 2012;40:D930-4.

28. National Human Genome Research Institute. The ENCODE Project: ENCyclopedia Of DNA Elements. http://www.genome.gov/encode/. Accessed 19 June 2015.

29. Blanco J, Muriel-Bombín A, Sagredo V, Taboada F, Gandía F, Tamayo L, et al. Incidence, organ dysfunction and mortality in severe sepsis: a Spanish multicentre study. Crit Care. 2008;12:R158.

30. Cho HY. Genomic structure and variation of nuclear factor (erythroidderived 2)-like 2. Oxid Med Cell Longev. 2013;2013:286524.

31. Yavartanoo M, Choi JK. ENCODE: a sourcebook of epigenomes and chromatin language. Genomics Inform. 2013;11:2-6.

32. Halliwell B, Gutteridge JM, Cross CE. Free radicals, antioxidants, and human disease: where are we now? J Lab Clin Med. 1992;119:598-620.

33. Lovat R, Preiser JC. Antioxidant therapy in intensive care. Curr Opin Crit Care. 2003;9:266-70.

34. Macdonald J, Galley HF, Webster NR. Oxidative stress and gene expression in sepsis. Br J Anaesth. 2003:90:221-32.

35. Victor VM, Rocha M, De la Fuente M. Immune cells: free radicals and antioxidants in sepsis. Int Immunopharmacol. 2004;4:327-47.

36. Winterbourn CC, Buss IH, Chan TP, Plank LD, Clark MA, Windsor JA. Protein carbonyl measurements show evidence of early oxidative stress in critically ill patients. Crit Care Med. 2000;28:143-9.

37. Sun ZT, Yang CY, Miao LJ, Zhang SF, Han XP, Ren SE, et al. Effects of mechanical ventilation with different tidal volume on oxidative stress and antioxidant in lung. J Anesth. In press. doi:10.1007/s00540-014-1954-z.

38. Cho HY, Reddy SP, Debiase A, Yamamoto M, Kleeberger SR. Gene expression profiling of NRF2-mediated protection against oxidative injury. Free Radic Biol Med. 2005;38:325-43.

39. Yang F, Coalson JJ, Bobb HH, Carter JD, Banu J, Ghio AJ. Resistance of hypotransferrinemic mice to hyperoxia-induced lung injury. Am J Physiol. 1999;277:L1214-23.

40. Gebel S, Gerstmayer B, Bosio A, Haussmann HJ, Van Miert E, Müller T. Gene expression profiling in respiratory tissues from rats exposed to mainstream cigarette smoke. Carcinogenesis. 2004;25:169-78.

41. Arcaroli JJ, Hokanson JE, Abraham E, Geraci M, Murphy JR, Bowler RP, et al. Extracellular superoxide dismutase haplotypes are associated with acute lung injury and mortality. Am J Respir Crit Care Med. 2009;179:105-12.

42. Reddy AJ, Christie JD, Aplenc R, Fuchs B, Lanken PN, Kleeberger SR. Association of human $\mathrm{NAD}(\mathrm{P}) \mathrm{H}$ :quinone oxidoreductase 1 (NQO1) polymorphism with development of acute lung injury. J Cell Mol Med. 2009;13:1784-91.

43. Chanock SJ, Manolio T, Boehnke M, Boerwinkle E, Hunter DJ, Thomas G, et al. Replicating genotype-phenotype associations. Nature. 2007;447:655-60.

44. Hindorff LA, Sethupathy P, Junkins HA, Ramos EM, Mehta JP, Collins FS, et al. Potential etiologic and functional implications of genome-wide association loci for human diseases and traits. Proc Natl Acad Sci U S A. 2009;106:9362-7.

\section{Submit your next manuscript to BioMed Central and take full advantage of:}

- Convenient online submission

- Thorough peer review

- No space constraints or color figure charges

- Immediate publication on acceptance

- Inclusion in PubMed, CAS, Scopus and Google Scholar

- Research which is freely available for redistribution 